\title{
Simple Groups with Prescribed Local Structure
}

\author{
ORAZIo PUGLISI
}

\section{Introduction.}

In recent years the construction of simple groups with local structure of prescribed type has been investigated in some works of Dixon, Evans and Smith ([DES1], [DES3]), while the problem of embedding a given group into a simple group whose local structure is, to some extent, known, was considered in [DES2]. These papers provide methods to construct simple groups which are locally-(P-by-finite), when $\mathcal{P}$ is some relevant group-theoretical property (e.g. solubility, nilpotence, residual finiteness etc..) and embedding theorems for some classes of groups are proved. In this short note we describe an alternative construction which yelds the same kind of results, using a more direct approach. Our approach works for groups of any infinite order, so that the restrictions on cardinalities needed in [DES1] and [DES3] can be dropped. This note is based upon a generalization of an idea developed in $[D]$.

\section{Simple locally-(P-by-finite) groups.}

When $\mathcal{P}$ is a group-theoretical property, we say that the group $G$ is $\mathcal{P}$ by-finite if $G$ has a proper and non-trivial normal $\mathcal{P}$-subgroup $N$ of finite index.

We say that a set $\mathcal{F}$ of subgroups of the infinite group $G$ is a directed residual system if its members are normal subgroups of finite index, $\bigcap\{H \mid H \in \mathcal{F}\}=1$ and, for every $H, K \in \mathcal{F}$ there exists $L \in \mathcal{F}$ such that $L \leq H \cap K$.

Indirizzo dell'A.: Dipartimento di Matematica “U. Dini”, Università di Firenze, Viale Morgagni 67A, I-50134 Firenze, Italy.

E-mail:puglisi@math.unifi.it 
Let $G$ be any infinite group. We shall consider $G$ embedded into $S=\operatorname{Sym}(G)$ via the right regular representation. Let $\mathcal{F}$ be a set of subgroups of $G$ and assume that, given $H, K \in \mathcal{F}$, there exists $L \in \mathcal{F}$ such that $L \leq H \cap K$. It is clear that, under this assumption, the set $M(\mathcal{F})=\bigcup_{H \in \mathcal{F}} C_{S}(H)$ is a subgroup of $S$. The group $M$ has been studied in [D], when $G=Z$ and $\mathcal{F}=\{H \leq Z \mid H \neq 0\}$. We call $M(\mathcal{F})$ the group of $\mathcal{F}$-modular permutations on $G$.

The set $\mathcal{L}=\left\{C_{S}(H) \mid H \in \mathcal{F}\right\}$ is a directed local system of $M(\mathcal{F})$, and the next lemma describes the structure of each $C_{S}(H)$.

Lemma 2.1. Let $G$ be embedded into $S=\operatorname{Sym}(G)$ via the right regular representation $\rho$. Choose $H$ any normal subgroup of $G$ of finite index $n$. Then $C_{S}(H) \simeq H \mathrm{wrS}_{n}$ and $G$ embeds into $C_{S}(H)$.

Proof. Choose a left transversal $T=\left\{t_{1}, \ldots, t_{n}\right\}$ for $H$ in $G$ and, for every $\sigma \in S_{n}$ define $\bar{\sigma} \in S$ by setting $\left(t_{i} h\right) \bar{\sigma}=t_{i \sigma} h$ for all $h \in H$. The set $\bar{S}_{n}=\left\{\bar{\sigma} \mid \sigma \in S_{n}\right\}$ is a subgroup of $C_{S}(H)$ isomorphic to $S_{n}$. Choose $x \in H$ and $t_{i} \in T$. The map $\tau_{i, x}$ defined by $\left(t_{j} h\right) \tau_{i, x}=t_{j} h$ when $j \neq i$ and $\left(t_{i} h\right) \tau_{i, x}=t_{i} x h$, is easily seen to be in $C_{S}(H)$. The function $\theta_{i}: H \longrightarrow C_{S}(H)$ sending $x$ to $\tau_{i, x^{-1}}$ is an injective group homomorphism. Moreover $B=\left\langle H^{\theta_{i}} \mid i=1, \ldots, n\right\rangle$ is isomorphic to the direct power of $n$ copies of $H$, since $\left[H^{\theta_{i}}, H^{\theta_{j}}\right]=1$ whenever $i \neq j$. The group $B$ is contained in the setwise stabilizer of the $H$-orbits $\left\{t_{i} H \mid i=1, \ldots n\right\}$. Conversely choose $\eta \in C_{S}(H)$ stabilizing the cosets of $H$ and, for each $i$, define $x_{i} \in H$ by $\left(t_{i}\right) \eta=t_{i} x_{i}{ }^{-1}$. A generic element of $G$ can be written uniquely as $t_{i} h$ for suitable $t_{i} \in T$ and $h \in H$. Thus

$$
\left(t_{i} h\right) \eta=\left(t_{i}\right) h^{\rho} \eta=\left(t_{i}\right) \eta h^{\rho}=\left(t_{i} x_{i}^{-1}\right) h^{\rho}=t_{i} x_{i}{ }^{-1} h
$$

Hence $\eta=\prod_{i=1}^{n}\left(x_{i}\right)^{\theta_{i}}$, proving that $B$ is the setwise stabilizer of the $H$-orbits.

The $H$-orbits are preserved by $C_{S}(H)$; hence, for every $\alpha \in C_{S}(H)$, $\left(t_{i} H\right) \alpha$ is still a coset of $H$. Let $\sigma \in S_{n}$ be such that $\left(t_{i} H\right) \alpha=t_{i \sigma} H$. The permutation $\bar{\sigma}^{-1} \alpha$ stabilizes all the $H$-cosets, hence $\bar{\sigma}^{-1} \alpha=b$ for a suitable $b \in B$. Whence $C_{S}(H)=\bar{S}_{n} B$ and the group $\bar{S}_{n} B$ is isomorphich to the permutational wreath product $H \mathrm{wr} S_{n}$ with $S_{n}$ acting naturally. Finally we recall that the image of the left regular representation $\lambda$ of $G$, is the centralizer in $S$ of $G^{\rho}$, whence $G^{\lambda} \leq C_{S}(H)$.

The following lemma contains some easy facts which can be found, in a slightly more general setting, in [DES2]. We give a proof for the reader's convenience. 
LEMma 2.2. Let $H$ be any group, $G=H \mathrm{wrS}_{n}$ a permutational wreath product where $n \geq 7$ and $B$ the base group of $G$. Then

1. $W=G^{\prime}=\left[B, A_{n}\right] A_{n}=\left(H \mathrm{wr} A_{n}\right)^{\prime}$;

2. if $x \in W \backslash\left[B, A_{n}\right]$ then $x^{W}=W$.

Proof. The base group $B$ is the direct product $H_{1} \times \ldots \times H_{n}$ of $n$ copies of $H$. If we define $K_{i}=\left\{\left(h_{1}, \ldots, h_{n}\right) \mid h_{j}=1 \forall j \neq i\right\}$, the subgroup $B$ can be written as $B=\prod_{i=1}^{n} K_{i}$. Choose $x, y \in H, \sigma=(123), \tau=(132) \in A_{n}$ and set $u=\left(x^{-1}, 1, \ldots, 1\right), v=\left(y^{-1}, 1, \ldots, 1\right)$. Then $[[u, \sigma],[v, \tau]]=([x, y], 1, \ldots, 1)$, proving that $K_{1}^{\prime} \leq\left[B, A_{n}\right] \leq\left[B, S_{n}\right]$. In particular $B^{\prime} \leq\left[B, A_{n}\right]$. Since $G^{\prime}=B^{\prime}\left[B, S_{n}\right] A_{n}$ we get $G^{\prime}=\left[K_{1}, S_{n}\right] A_{n}$. For any given $\sigma \in S_{n}$, there exists $\tau \in A_{n}$ such that $1 \sigma=1 \tau$. Hence, for all $k \in K_{1}$, one has $[k, \sigma]$ $=k^{-1} k^{\sigma}=k^{-1} k^{\tau}=[k, \tau]$, showing that $\left[K_{1}, S_{n}\right]=\left[K_{1}, A_{n}\right]$. Therefore $W=\left[K_{1}, A_{n}\right] A_{n}=\left[B, A_{n}\right] A_{n}=\left(H \mathrm{wr} A_{n}\right)^{\prime}$, proving (1).

In order to prove (2) choose $r, s \in H$, set $u=\left(r^{-1}, r, 1, \ldots, 1\right)$, $v=\left(s^{-1}, 1, s, 1, \ldots, 1\right)$ and consider the even permutations $\sigma=(14)(25)$, $\tau=(16)(37)$. The quotient $W /\left[B, A_{n}\right]$ is simple, hence $W=x^{W}\left[B, A_{n}\right]$ and we can find $a, b \in\left[B, A_{n}\right]$ such that $\sigma a, \tau b \in x^{W}$. Since $u, v$ are in $W$, the commutator $[[u, \sigma a],[v, \tau b]]$ belongs to $x^{W}$. On the other hand the elements $u^{\sigma a}$ and $v^{\tau b}$ have support $\{4,5\}$ and $\{6,7\}$ resp., so that they commute and centralize both $u$ and $v$. Thus $[[u, \sigma a],[v, \tau b]]=\left[u^{-1}, v^{-1}\right]=([r, s], 1 \ldots, 1)$, showing that $K_{1}^{\prime} \leq x^{W}$. Since $K_{1}^{\prime}$ is normalized by $B$, we have

$$
\left(K_{1}^{\prime}\right)^{x^{W}}=\left(K_{1}^{\prime}\right)^{\left[B, A_{n}\right] x^{W}}=\left(K_{1}^{\prime}\right)^{\left[B, A_{n}\right] A_{n}}=\left(K_{1}^{\prime}\right)^{A_{n}}=B^{\prime} .
$$

Thus $B^{\prime} \leq x^{W}$ and, to complete the proof, we may assume $H$ to be abelian. The group $A_{n}$ stabilizes the series $\left[B, A_{n}, A_{n}\right] \leq\left[B, A_{n}\right] \leq B$. On the other hand $A_{n}$ is simple because $n \geq 7$, hence $\left[B, A_{n}, A_{n}\right]=\left[B, A_{n}\right]$. The group $W / x^{W}=x^{W}\left[B, A_{n}\right] / x^{W}$ is isomorphic to a quotient of $\left[B, A_{n}\right]$ so that is abelian. But $W^{\prime}=\left[B, A_{n}, A_{n}\right] A_{n}=\left[B, A_{n}\right] A_{n}=W$, hence $W$ is perfect and has no non-trivial abelian images. Thus $x^{W}\left[B, A_{n}\right]=x^{W}$, proving (2).

We can now extend Theorem 2 of [DES2].

THEOREM 2.3. Let $G$ be any residually finite group. There exists a simple group $S$ such that

1. $G^{\prime}$ embeds in $S$;

2. each finitely generated subgroup of $S$ is isomorphic to a finite extension of a subgroup of a finite direct power of $G$;

3. $|S|=|G|$. 
Proof. Let $\mathcal{F}$ be any directed residual system. Without loss of generality we may assume that $G / H$ has order at least 7 when $H$ belongs to $\mathcal{F}$. Since $\mathcal{F}$ is directed, $M(\mathcal{F})=\bigcup_{H \in \mathcal{F}} C_{\operatorname{Sym}(G)}(H)$ is a group. Moreover, by lemma 2.1, each $C_{\mathrm{Sym}(G)}(H)$ is isomorphic to $H \mathrm{wr} S_{n}$ where $n=|G: H|$. We prove that $S(\mathcal{F})=M(\mathcal{F})^{\prime}$ is simple. For $H \in \mathcal{F}$ write $C_{\mathrm{Sym}(G)}(H)=B_{H} S_{H}$ where $S_{H}$ is the symmetric group of degree $|G: H|$ and $B_{H}$ indicates the base group of $H \mathrm{wr} S_{H}$. It is useful to notice that, if $A_{H}$ is the alternating subgroup of $S_{H}$, we have

$$
S(\mathcal{F})=\bigcup_{H \in \mathcal{F}}\left(C_{\operatorname{Sym}(G)}(H)\right)^{\prime}=\bigcup_{H \in \mathcal{F}}\left[B_{H}, A_{H}\right] A_{H} .
$$

Since $G$ embeds in $M(\mathcal{F})$ via the left regular representation, $G^{\prime}$ embeds into $S(\mathcal{F})$. Let $N$ be a normal subgroup of $S(\mathcal{F})$. Two cases can occur.

1. For each $K \in \mathcal{F}$, there exists $H \in \mathcal{F}$ with $H \leq K$ and $N \cap\left[B_{H}, A_{H}\right] A_{H}$ not contained in $\left[B_{H}, A_{H}\right]$.

Let $r \in S(\mathcal{F})$ be any element, and choose $K$ such that $r \in\left[B_{K}, A_{K}\right] A_{K}$. Select $H \in \mathcal{F}$ in such a way that $H \leq K$ and $N \cap\left[B_{H}, A_{H}\right] A_{H}$ is not contained in $\left[B_{H}, A_{H}\right]$. It is then possible to find $x \in N \cap\left[B_{H}, A_{H}\right] A_{H} \backslash\left[B_{H}, A_{H}\right]$. By lemma 2.2, $x^{\left[B_{H}, A_{H}\right] A_{H}}=\left[B_{H}, A_{H}\right] A_{H}$ hence $r \in x^{\left[B_{H}, A_{H}\right] A_{H}} \leq x^{S(\mathcal{F})} \leq N$. Since this holds for every $r$, we have that $N=S(\mathcal{F})$.

2. For each $K \in \mathcal{F}, N \cap\left[B_{K}, A_{K}\right] A_{K} \leq\left[B_{K}, A_{K}\right]$.

Let $H$ be in $\mathcal{F}$ and choose $x \in N \cap\left[B_{H}, A_{H}\right] \leq B_{H}$. If $x$ is not the identity, there exists $r \in G$ such that $(r) x \neq r$. The set $\mathcal{F}$ is a directed residual system, so that we can find $L \in \mathcal{F}$ such that $L \leq H$ and $r L \neq(r) x L$. Since $B_{H} A_{H} \leq B_{L} A_{L}$, the element $x$ belongs to $\left[B_{L}, A_{L}\right]$ so that $x$ normalizes the $L$-cosets. On the other hand, for any given $a \in L$, $(r a) x=\left(r a^{\rho}\right) x=(r) a^{\rho} x=(r) x a^{\rho}=(r) x a$. Thus $(r L) x=(r) x L$ and this contradicts the fact that $x$ normalizes the left $L$-cosets. Hence $N \cap\left[B_{H}, A_{H}\right] A_{H}=1$ for every $H \in \mathcal{F}$, showing that $N=1$.

Since $\mathcal{F}$ has cardinality at least $\kappa=|G|$, the group $S(\mathcal{F})$ has cardinality at least $\kappa$. By proposition 3.1 of [DES3], $G^{\prime}$ is contained in a simple subgroup $S$ of $S(\mathcal{F})$, of cardinality $\kappa$. It is clear that $S$ has the claimed properties.

COROLLARY 2.4. Let $\mathcal{P}$ be a group-theoretical property closed under taking direct products and subgroups. If there exists an infinite residually-finite $\mathcal{P}$-group then, for every infinite cardinal $\kappa$, there exists a simple group $S(\kappa)$ of cardinality $\kappa$, with a local system of $\mathcal{P}$-by-finite subgroups. 
Proof. Suppose there exists an infinite residually finite $\mathcal{P}$-group $R$. There is no loss of generality in assuming that $R$ is countable. Given an infinite cardinal $\kappa$ let $G$ be the direct product of $\kappa$ copies of $R$. The group $G$ is a residually finite $\mathcal{P}$-group of cardinality $\kappa$. Apply theorem 2.3 to $G$ to construct a simple group $S(\kappa)$ of cardinality $\kappa$, with a local system of $\mathcal{P}$-byfinite subgroups.

Also theorem 1 of [DES2] can be generalized to groups of any infinite cardinality.

THEOREM 2.5. Let $\mathcal{B}$ be a variety in which the free groups of finite rank are residually finite. For every cardinal $\kappa$ there exists a simple group $S(\kappa)$ such that

1. $S(\kappa)$ is locally-(B-by-finite);

2. $S(\kappa)$ has cardinality $\lambda=\max \left\{\kappa, \aleph_{0}\right\}$;

3. $S(\kappa)$ contains a copy of $V(\kappa)$, the $\mathcal{B}$-free group of rank $\kappa$.

Proof. If $\kappa$ is an infinite cardinal, the group $V(\kappa)$ is residually-( $\mathcal{B}$-free of finite rank), and then $V(\kappa)$ is residually-finite. Thus $V(\kappa)$ is residuallyfinite for every cardinal $\kappa$, finite or infinite. Let $C$ be a cyclic group of order 2 and set $G=V(\kappa)$ wr $C$. The group $G$ is residually finite and has cardinality $\lambda=\max \left\{\kappa, \aleph_{0}\right\}$. It is readily seen that $[B, C]$ has an image isomorphic to $V(\kappa)$ so that $V(\kappa)$ embeds into $[B, C] \leq G^{\prime}$. Theorem 2.3 can now be invoked to get the claim.

\section{REFERENCES}

[D] F. Del Castillo, Modular permutations on Z, Rend. Sem. Mat. Univ. Padova, 118 (2007), pp. 147-158.

[DES1] M. R. Dixon - M. J. Evans - H. SMith, Embedding groups in locally (soluble-by-finite) simple groups, J. Group Theory, 9 (2006), pp. 383-395.

[DES2] M. R. Dixon - M. J. Evans - H. SMith, Simple groups with prescribed local properties, J. Group Theory, 12 (2009), pp. 745-752.

[DES3] M. R. Dixon - M. J. Evans - H. Smith, Some simple locally (soluble-byfinite) groups, pp. 79-89 in Ischia Group Theory 2008, M. Bianchi, P. Longobardi, M. Maj and C. M. Scoppola (eds.), World Scientific (2009).

Manoscritto pervenuto in redazione il 9 giugno 2010. 